\title{
Key Algorithm of 3D Streaming Storage in Cloud
}

\author{
Ge Yang* and Yang Ding \\ Engineering Lab on Intelligent Perception for Internet of Things (ELIP), Shenzhen Graduate School, Peking University, Shenzhen \\ 518055, China \\ * Corresponding author
}

\begin{abstract}
According to the cloud-p2p storage system model, cloud environment 3D streaming media storage algorithm is proposed. It includes 3DVSCP (3D video storage based on cloudp2p algorithm) and UDCP (user on demand based on the cloudp2p algorithm).3DVSCP algorithm to add video to reduce the load of the server, while providing adequate video copy of the user's demand for the future. Experiments show that the server storage capacity occupied by, the 3DVSCP algorithm than in the UQSS (user quota storage system) HybSim (hybrid streaming media storage and mixed flow storage media) algorithm can save $70 \%$ to $80 \%$; In the user request response rate and response with peer to peer structure (MingCloud) CAPVDD (cloud assisted P2P video on demand distribution, cloud assisted P2P VOD distribution) algorithm, compared with faster and more stable characteristics.
\end{abstract}

Keywords-cloud storage; P2P (Peer-to-peer) network; 3D streaming media; cloud-P2P storage model

\section{INTRODUCTION}

The cloud computing, as a new type of basic service facilities, has been fully popularized with the further research and rapid development. At the same time, as an important component in cloud computing and extended concept, cloud storage is an great innovation and transformation of storage industry technology and services ${ }^{[1]}$

In order to solve the problem of heavy load of the server, the related literatures put forward the technology P2P streaming media transmission which uses the resources of peer nodes to provide services for other nodes. But the technology will occupy the client's resources too much, the client terminal has a higher performance, meanwhile, there is no effective solution to the problem of server bottlenecks and massive sea view data storage..

\section{RELATED WORK}

\section{A. 3D Streaming Media}

3D video also contains color information and depth information, which leads to the 3D streaming media needs a huge redundant data. Moreover, 3D video also need to have greater information Due to the limited bandwidth, the storage and transmission of the video playback quality problem causes a lot of problems, and people have to face the problems that downloading the 3D video file which has large memory in such a slow network speed required a lot of time and 3D video screen showing fuzzily ${ }^{[2]}$.
By now, one of the most popular 3D video transmission technology is the frame symmetric drop algorithm which proposed in the literature ${ }^{[3]}$. It means that any video frame must be fully buffered before the last time it is played in order to ensure that the video is played in real time. The implementation method is to divide the video into several groups and selectively discard some video frames for reducing the redundancy in video transmission. But the algorithm does not apply to video transmission.

\section{B. Cloud-P2P System Model}

Cloud-P2P is a fusion system model of cloud computing and $\mathrm{P} 2 \mathrm{P}$ technology. The server unified scheduling and providing the basis of streaming media on demand service to ensure that users watch the video smoothly. At the same time, make full use of the available resources on the P2P network node, transferring the streaming media on demand task submitted by some users from the network center to the P2P network user node, so as to reduce the workload of the server. In summary, this paper tries to put forward the 3DVSCP algorithm and UDCP algorithm based on a server cluster and the edge node of the network composed of the broader public virtual resource pool.

In this paper, we put forward a combination of cloud storage and streaming media technology and a storage model based on Cloud-P2P system which based on the Hadoop cloud computing platform and combined with the cloud storage technology. The 3DVSCP algorithm and UDCP algorithm, which is based on the popular degree of the stream media and the user level, is used to solve the problem of decreasing the user satisfaction which is caused by the load on the server side and the slow speed of the user's response.

\section{DVSCP ALGORITHM}

In order to ensure the users to watch the $3 \mathrm{D}$ streaming media, the three-dimensional image of the right and left eye perception needs to be obtained simultaneously. At the same time, to applicate a video directory node for the video, and set the parameters of the initial value. In this paper, in order to distinguish between high prevalence documents and low popularity documents, set the variable parameters: the popularity threshold. The popularity of streaming media files over the threshold is high popularity files, the opposite is low popularity files.

Symbols and formulas:

- $\quad$ I: Represents the ith 3D video file 
- $\mathrm{N}$ : Represents the total number of video files in the current video directory

- $\mathrm{T}_{\mathrm{i}}$ : Represents the ith $3 \mathrm{D}$ video's play time in a day

- $\mathrm{H}_{\mathrm{i}}$ : Represents the ith 3D video's number of on demand in a day

- $\mathrm{P}_{\mathrm{i}}$ : Represents the ith 3D video's popularity

- $\mathrm{I}_{\mathrm{i}}$ : Represents the ith 3D video's release time

- $\mathrm{Q}_{\mathrm{i}}$ : Represents the ith node's weight at the same day

In this paper, the concept of a video weight is proposed, which is determined by the time difference between the video release time and the current time. With the emergence of genuine video on the network, the popularity of the video will get a peak. And then the video popularity will have a huge fall. Finally, with the passage of time, the video popularity is basically stable. Therefore, it can be said that the video weight is subject to the time difference of the Poisson distribution, and we can get the linear regression formula of video weight:

$$
\mathrm{Q}_{\mathrm{i}}=\left(\lambda^{\mathrm{Ii}}\right) *\left(\mathrm{e}^{-\lambda}\right) /\left(\mathrm{I}_{\mathrm{i}} !\right)
$$

The monitoring module counts the length and the ordering number of streaming file, and calculates the total time the users watch streaming vedio within a day and the total number of on demand of streaming media files within a day in the system. Combine the expression (1) the popularity of video $i$ is:

$$
\mathrm{P}_{\mathrm{i}}=\left(\mathrm{T}_{\mathrm{i}} * \mathrm{H}_{\mathrm{i}}\right) /\left(\sum_{\mathrm{i}=1}^{\mathrm{N}} \mathrm{Ti} * \sum_{\mathrm{i}=1}^{\mathrm{N}} \mathrm{Hi}\right)^{*} \mathrm{Q}_{\mathrm{i}}
$$

Expression (2) indicate that the larger the $\mathrm{Pi}$ is, the greater the user's attention to the streaming media file $i$, that is, the streaming media file I will have more people to visit for a long time, and the number of clicks is also more.

\section{UDCP ALGORITHM}

In order to ensure the effect of stereo video, we must play a number of frames at the same time and solve the heterogeneous problem. The solution proposed in this paper is to ensure that the stream media data can be quickly reached to the user side by blocking the bandwidth of the load. At the same time, in order to guarantee the efficiency of the network transmission, the data transmission is carried out by the layer.

\section{Symbol description:}

- $\alpha$ : Represents the security point of the server load, can be set as $0.2 \sim 0.5$

- $\quad \beta$ : Represents the risk point of the server load, can be set as $0.7 \sim 0.8$

- $\mathrm{x}$ : Represents the current server load

\section{Arithmetic statement:}

- Users access and query the server video storage directory;
- The user selects the video content and sends the request;

- Server testing system to query the current load situation: if the server's load is currently in the degree of $\mathrm{X}$ load $(0, \alpha]$ interval, the service request directly to the server to join the queue, go to 6 ; If the server is currently loaded with $\mathrm{X}$ in the $(\alpha, \beta]$ interval, go to 4 ; If the current server load in $X(\beta,+\infty)$ interval, to 5 ;

- Further detection of the user level, if the user is advanced and intermediate users, the service request to join the server queue, to 6 ; Otherwise, the popularity of the video on demand is further detected, if the 3D video' popularity is low, the service request will be added to the server service queue, to 6; Otherwise, the service request is added to the replica node service queue, to 7 ;

- To detect the user's level, if the user is an senior user, the service request is added to the server queue, go to 6 ; Otherwise, the popularity of the video on demand is further detected, if the $3 \mathrm{D}$ video is low, the service request will be added to the server service queue, go to 6; Otherwise, the service request is added to the replica node service queue, go to 7 ;

- The service request is deployed to the corresponding server node to complete the follow-up service request response;

- The service request is deployed to the corresponding user node to complete the follow-up service request response.

\section{SIMULATION EXPERIMENT}

\section{A. Simulation Experiment Environment}

In this paper, the Zip-f distribution is based on the simulation of the popularity of each stream media, in which the Zip-f simulation parameters $\alpha$ is $0.271^{[4]}$; By random number simulation of the user on demand, including the user demand time and on-demand streaming media ID; By numerical experiments, we can find that the online time of the copy of the user node is in the exponential distribution of the $\mathrm{S}=0.5^{[5]}$, which can simulate the online situation of the user node. In this experiment, the required parameters and settings are as follows:

- Node availability. That is a copy of the peer node online. A copy of the node's online total with its online time, user level and distance from the last on-line, the higher the level and distance from the last on-line time is longer, the greater the probability of the user online.

- Copy number of 3D streaming media files with high popularity. It is found that, when the copy is less, with the increase of the number $\mathrm{H}$ of copies, the probability of the user data request served by the replica node of node storage layer is increased, and the transmission rate $\beta \mathrm{c}$ of the node storage layer is increased $\mathrm{d}^{[5]}$. As the number of copies increases to a certain value, with the 
increase of the number $\mathrm{H}$ of copies, the increased tendency of the transmission rate $\beta \mathrm{c}$ of the node storage layer is to slow down, and the trend is gradually close to $100 \%$.

- In this paper, the popular threshold value is 0.005 .

- In this paper, the security point of the server's performance is 0.3 , and the risk point is 0.7 .

- The total number of users on demand, that is, the number of requests for a number of users in a certain period of time.

- The storage capacity of the server and the user nodes, that is, the storage capacity of the streaming media file that the server node and the user node are used to store, which is the important data base of the 3DVSCP algorithm and HybSim algorithm ${ }^{[6]}$.

- The user request response rate and the user request response are the important parameters to compare the processing efficiency of the UDCP algorithm with the MingCloud's user demand in the cloud storage. The user request response rate is the delay time from the user's request to implement the request, including data transmission delay and data processing delay; the user request response degree is the ratio of the number of user requests in the same time period and the system satisfies the number of users' requests.

\section{B. Simulation Experiment Results and Analysis}

The storage (GB) of the server nodes, four groups of experiments were carried out in this experiment. The corresponding experiments are carried out by changing the number of streaming media resources with different popularity threshold by the monitoring module of the system of monitoring and statistics, such as table 1 and table 2 .

TABLE I. 3DVSCP ALGORITHM SERVER COPY OF THE STORAGE (FIRST SETS OF PARAMETERS)

\begin{tabular}{ccccccc}
\hline Video Number & \multicolumn{1}{c}{100} & 200 & 300 & 400 & 500 \\
\hline popularity is 0.05 & 553.8 & 806 & 1021.8 & 1314.2 & 1395.64 \\
\hline
\end{tabular}

TABLE II. HYBSIM ALGORITHM SERVER COPY OF THE STORAGE (FIRST SETS OF PARAMETERS)

\begin{tabular}{cccccc} 
Video Number & 100 & 200 & 300 & 400 & 500 \\
\hline popularity is 0.05 & 953 & 1550 & 2409 & 3283 & 4126
\end{tabular}

The experimental data of the table 1 and table 2 can prove clearly that the 3DVSCP algorithm proposed in this paper compared to the HybSim algorithm has a lot of optimization of storage utilization. As for the different number of streaming media of different popularity threshold, the corresponding optimized ratio is the server copy storage in the DVSCP algorithm divided by he server copy storage in the HybSim algorithm, as shown in figure 1 :

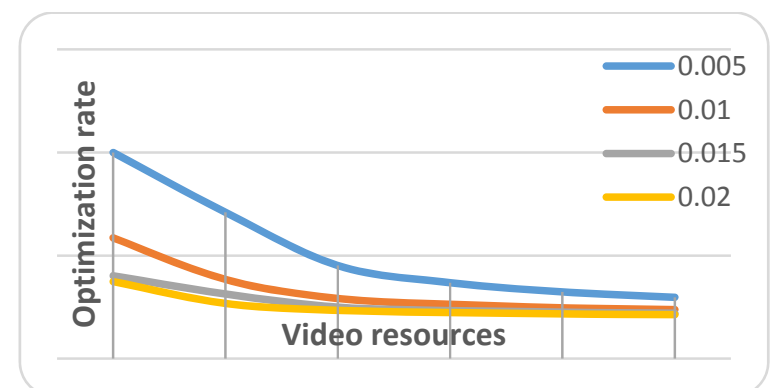

FIGURE I. THE OPTIMAL RATE OF 3DVSCP ALGORITHM AND HYBSIM ALGORITHM FOR STREAMING MEDIA COPIES OF THE STREAMING MEDIA IN THE SERVER SIDE

Figure 1 shows that compared to the HybSim algorithm, the 3DVSCP algorithm can save a lot of server storage space, and with the increase of the number of video resources, the savings will be more obvious. From Figure 1,we can see that when the number of video resources is greater than or equal to 200 , the 3DVSCP algorithm in this paper can save about $60 \% \sim 80 \%$ of memory storage.

Then, the UDCP algorithm proposed in this paper is analyzed and compared with the CAPVDD algorithm under MingCloud [7].

Assuming that there are $20,50,80$ or 100 users on demand in one hour, according to the number of different resources, calculate and analyze the response rate of the two systems. Recording the time of the user on demand and the time of the streaming media began to play, and the time difference of the two time is the buffer time of the streaming media. In addition, according to the user's rating and on-demand video popularity to determine a weight of rate, the weight and time difference is multiplied by the system to respond to the user's request response rate, as shown in Figure 2:

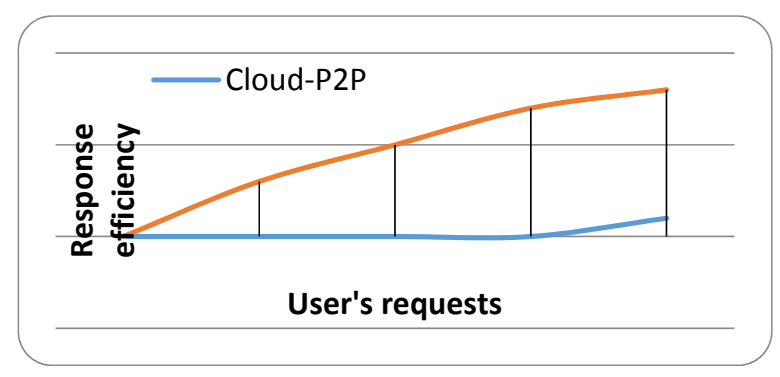

FIGURE II. THE RESPONSE RATE OF 20 USERS

You can see from Figure 2, while 20 user sends request in an hour, compared to the CAPVDD algorithm, the UDCP algorithm has faster response, and the response rate of UDCP algorithm is more stable. This is because when the user request is less, the UDCP algorithm will respond to the user request by the server side, so it is faster and more stable than the user request of the cloud storage model which fully utilizes the peer structure of the user node.

\section{SUMMARY}

In this paper, getting the relevant data by the simulation of the experimental data to so that the proposed 3DVSCP 
algorithm compares with the HybSim algorithm, and the UDCP algorithm compares with the CAPVDD algorithm. Experimental simulation data show that the 3DVSCP algorithm proposed in this paper is more optimized than the HybSim algorithm in terms of server storage and utilization, which can greatly reduce the burden of the server's copy storage; In response to the user request, compared to the CAPVDD algorithm, the UDCP algorithm can more quickly and efficiently respond to user requests.

\section{ACKNOWLEDGMENT}

This research was financially supported by Major Scientific Research Project for Universities of Guangdong Province (201612008QX); Guangdong Provincial special funds Project for Discipline Construction (No.2013WYXM0122); Guangdong Provincial College Innovation and Entrepreneurship Project (201613177024); Scientific Research Project of Shenzhen (JCYJ20160428153620486, JCYJ20170303140803747); Key Laboratory of Intelligent Multimedia Technology(201762005).

\section{REFERENCES}

[1] Wang Dewen, Liu Xiaomeng. Resource scheduling of cloud computing platform based on improved particle swarm optimization [J],.Application Research of Computers. 2015, 32(11):3230-3235.

[2] WANG Xiaomao, HUANG Chuanhe, ZHAO Jianhui, et al. Timedivision algorithm for converting planar video into 3D video based on inteligent scene analysis. Computer Engineering and Applications, 2012, 48(22) : 201-204.

[3] Chai Xuezhi, Cao jian. Cloud computing oriented workflow technology [J]. Journal of Chinese Computer Systems, 2012, 33(1):90-95.

[4] Yang Fuzheng, Wan Shuai. Overview of state-of-the-art and future of networked video quality assessment $[\mathrm{J}]$. Journal on Communications, 2016, 33(4):107-114.doi: 10.3969/j.issn.1000-436X.2012.04.015.

[5] Jiang Zhuoxuan, Zhuang yan, Li Xiaoming. Learning behavior analysis and prediction based on MOOC data [J]. Journal of Computer Research and Development, 2015, 52(3):614-628. doi:10.7544/issn10001239.2015.20140491.

[6] Jin Shunfu, Wang Chenfei, Chen Lingling, Huo Zhanqiang. Modeling and analysis of Cloud-P2P storage architecture [J]. Journal on Communication, 2015,36(3) 2015077-1-20150778.doi:10.11959/j.issn.1000-436x.2015077.

[7] Guo Hongfang, Lin Yusong, Wang Zongmin. Research on streaming distribution of cloud assisted $\mathrm{P} 2 \mathrm{P}$ video-on-demand $[\mathrm{J}]$. Application Research of Computers, 2013,30(2):526-534. doi:10.3969/j.issn.10013695.2013.02.057. 